\title{
Penetrating excimer Laser-assisted keratoplasty for corneal epithelial downgrowth in childhood
}

\author{
Barbora Majkutova $^{1 *}$, Fidelis Flockerzi ${ }^{2}$, Berthold Seitz M$^{3}$, Celestina Schober ${ }^{1}$ and Arne Viestenz ${ }^{1}$ \\ ${ }^{1}$ Department of Ophthalmology, Martin Luther University Halle-Wittenberg, Germany \\ ${ }^{2}$ Department of General and Special Pathology, Saarland University Hospital, UKS, Germany \\ ${ }^{3}$ Department of Ophthalmology, Saarland University Hospital, UKS, Germany
}

\begin{abstract}
Purpose: To report a case of excimer laser-assisted keratoplasty for corneal epithelial downgrowth after corneal laceration in childhood.

Methods: Report of a 5-year-old boy with history of corneal injury. The optical coherence tomography of the anterior segment as well as slit-lamp biomicroscopy were suspicious for epithelial downgrowth because of a corneal intrastromal white membranous structure.

Results: The patient underwent penetrating excimer laser-assisted keratoplasty 3 months after the injury. BCVA improved from 0.6 before surgery to 1.0 within 2 years of follow-up without any signs of acute inflammation or new epithelial downgrowth.

Conclusions: Epithelium can invade through a corneal wound to iris, trabecular meshwork and ciliary body after trauma or intraocular surgery. In extreme cases, migrates epithelium into the retina and under the choroid and may induce tractional retinal detachment. Lamellar epithelial downgrowth may lead to the melting of the corneal stroma. Early surgical intervention can prevent multiple of complications including loss of vision.
\end{abstract}

\section{Case Report}

A 5-year-old boy was presented in our department one month after corneal injury caused by metal stick, complaining of pain in his right eye and pronounced photosensitivity. The primary removal of corneal foreign body took place in an external clinic. BCVA was $0.6 \mathrm{OD}$ and 1.0 OS. The slit-lamp biomicroscopy examination revealed a corneal intrastromal whitish membranous structure in the right eye spreading over the visual axis (Figure 1).

The internal ocular structures were not affected. The left eye was completely normal.

By use of optical anterior segment coherence tomography an intrastromal scar formation and whitish intrastromal "membrane" were indentified, which appeared to be epithelial downgrowth (Figure 2).

On the ground of poor visual prognosis and incriminating discomfort a surgical intervention was recommended.

The patient underwent penetrating excimer laser-assisted keratoplasty (BS) three months after the injury. The histopathological

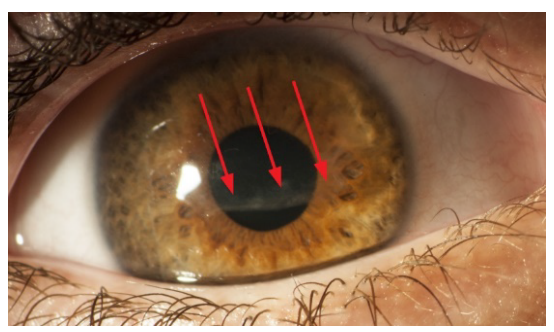

Figure 1. Slit lamp photograph of the right eye demonstrating intrastromal white membranous structure (arrows)

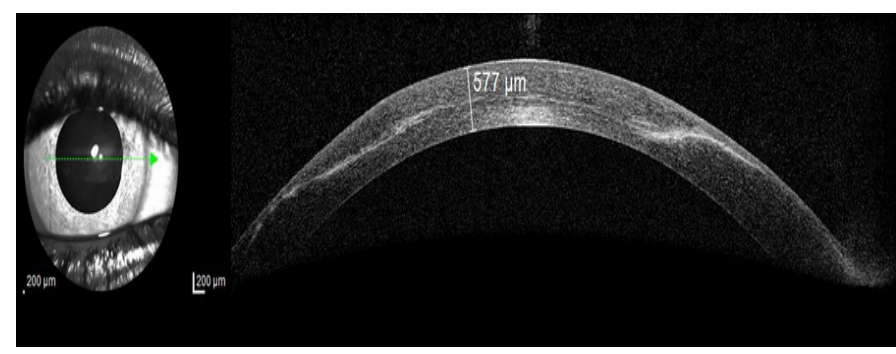

Figure 2. Optical anterior segment coherence tomography with intrastromal membrane

examination of the excised cornea showed downgrowth of stratified squamous epithelium through the Bowman's layer into the corneal stroma (Figure 3).

One-year after the intervention, the patient complained about impaired vision. Despite the treatment and keratoplasty the BCVA was $0.25 \mathrm{OD}$. The graft showed focal nonvascularized pannus adjacent to the double running sutures (Figure 4).

A corneal suture removal combined with superficial lamellar keratectomy had been performed.

${ }^{\star}$ Correspondence to: Barbora Majkutova, Department of Ophthalmology, University Clinic and Polyclinic for Ophthalmology, Martin Luther University Halle-Wittenberg, Germany, E-mail: majkutova.b@gmail.com

Key words: excimer laser keratoplasty, epithelial downgrowth, corneal graft, children

Received: May 07, 2020; Accepted: May 27, 2020; Published: May 29, 2020 
At 20 months of follow-up, after intensive local anti-inflammatory therapy, the BCVA improved up to $1.0 \mathrm{OD}$. The graft remained clear and showed no evidence of recurrence of epithelial downgrowth (Figure 5).

\section{Discussion}

Epithelial downgrowth (also termed epithelial ingrowth) is a vision-threatening complication, where epithelial cells invade into intraocular structures. It is a rare complication of ophthalmic surgery or penetrating ocular trauma [1].

There are several important factors for physiological corneal epithelial wound healing process, including the type and depth of

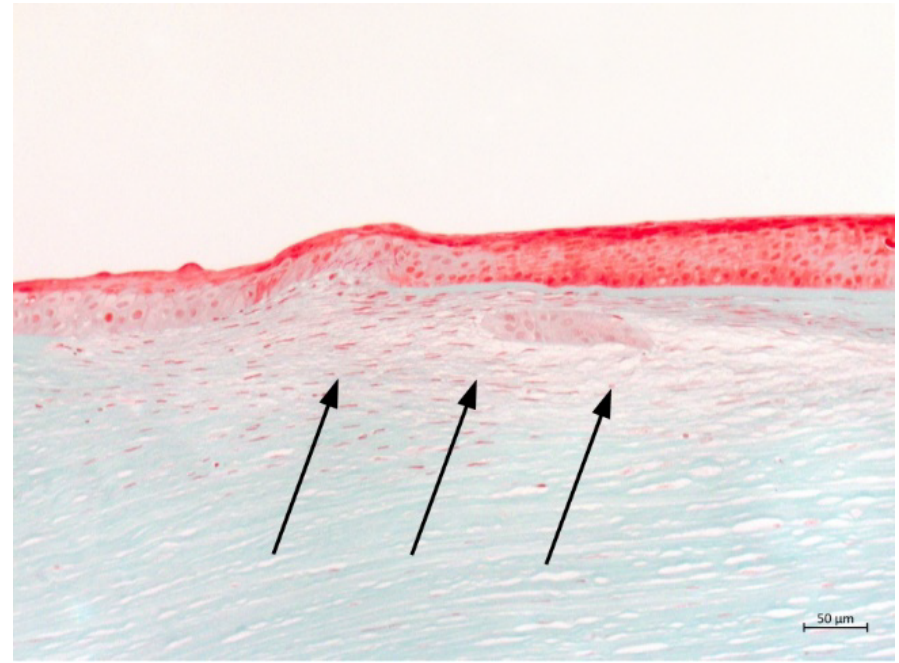

Figure 3. Histological image showing downgrowth of stratified squamous epithelium (arrows), (Masson-Goldner trichrome staining)

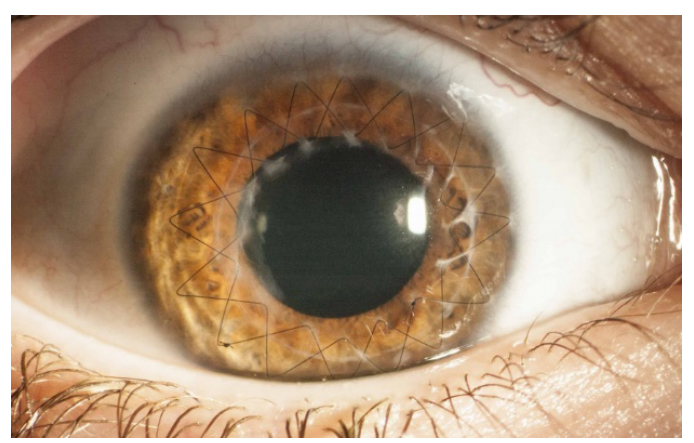

Figure 4. Corneal pannus adjacent to the double running suture one year after keratoplasty

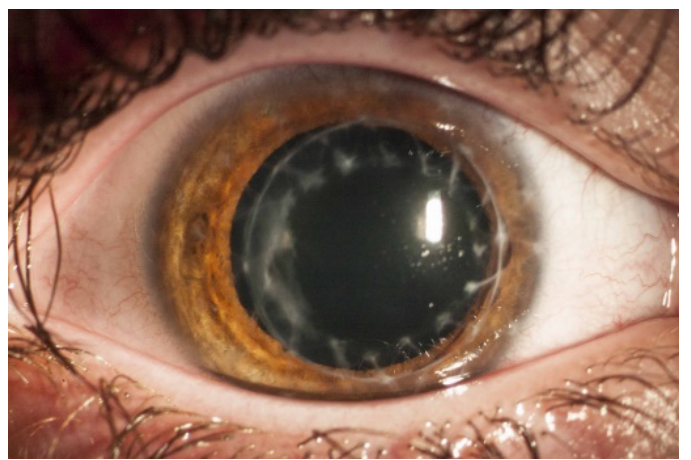

Figure 5. Right eye after suture removal and superficial lamellar keratectomy (BCVA 1.0) the injury, presence of inflammation, systemic disease, medication, neurotrophic keratopathy or potency of limbal stem cells.

Epithelial downgrowth is formed in a cystic, diffuse (sheets) or combined pattern. Proliferating cells that form an inclusion cyst connected to the wound occurs in cystic pattern, tends to have better prognosis [1]. In case of diffuse form, the epithelium can invade through the cornea to iris, trabecular meshwork and ciliary body and in extreme cases into the retina and under the choroid resulting in tractional retinal detachment. Furthermore, it can cause multiple other severe complications including secondary angle closure glaucoma, corneal decompensation, lens dislocation, astigmatism and visual impairment or blindness. Additionally, in our case corneal melting was one of the possible vision-threatening complications.

Several clinical features could be found in case of epithelial downgrowth. These include anterior chamber cells or iritis, glaucoma (unresponsive to conventional therapy), posterior corneal and iris membrane [2]. If the diagnosis of epithelial downgrowth is questionable the optical coherence tomography of the anterior segment, confocal microscopy or ultrasound biomicroscopy (UBM) can be performed [3-7].

Cytokeratin as immunohistochemical marker is essential to confirm epithelial downgrowth [8].

Despite extensive attempts with various surgical and medical techniques by many investigators is the prognosis of epithelial downgrowth limited [1]. Therapeutic options (i.a.) consist of penetrating keratoplasty, en-block-excision with tectonic keratoscleroplasty [4,6,7], DALK (deep anterior lamellar keratoplasty), corneal abrasion with flaplifting, peeling and mitomycin $\mathrm{C}$ irrigation, corneal suture or bandage contact lens.

In any early intervention (lamellar or penetrating keratoplasty) it is essential to preserve the limbus area to provide the best visual outcome.

The downgrowth of surface epithelium is a disastrous but fortunately rare complication of intraocular surgery [2] or injury [1,4,6,7,9-11]. In the literature we can find several cases of epithelial downgrowth after trauma, which can lead years after the primary injury to loss of vision $[1,4,9,10,12]$. For this reason, an early and invasive intervention to achieve better visual outcome is required $[4,6,7,9]$.

In this case, one of the possible therapies of choice is deep anterior lamellar keratoplasty (DALK). This technique had been proved to be an efficient alternative to penetrating keratoplasty in several indications. Nevertheless, performing of DALK could be very challenging, especially when there is significant scaring of deep corneal stroma. Many surgeons may use penetrating keratoplasty as the primary procedure [13]. Among other possible complication, that we have to take into consideration, belong ruptures and microperforations of Descemet's membrane, as well as interface haze and irregularities [13] using DALK.

In our case, where we cannot surely exclude epithelial ingrowth in deep stroma or even in endothelium and Descemet's membrane, DALK would not be the therapy of choice.

With children as patients we have to consider not only longterm complications of epithelial downgrowth but also a complication caused by any intervention. Regarding to patient's age and future eye-development was in our case the therapy of choice penetrating keratoplasty with excimer laser [14]. 


\section{Conclusion}

In summary, we showed a case of child which presented only few months after corneal injury with severe complication (pain, photosensitivity, vision-impairment) as well as clinical features associated with epithelial downgrowth. To the best of our knowledge, we report for the first time of a case of epithelial downgrowth after corneal injury treated with excimer laser keratoplasty with promising outcome in childhood. Future follow-up and regular check-ups of our young patient are still necessary to confirm long-term results.

\section{References}

1. Weiner MJ, Trentacoste J, Pon DM, Albert DM (1989) Epithelial downgrowth: a 30year clinicopathological review. Br J Ophthalmol 73: 6-11.

2. Feder RS, Krachmer JH (1985) The diagnosis of epithelial downgrowth after keratoplasty. Am J Ophthalmol 99: 697-703.

3. Chiou AG, Kaufman SC, Kaz K, Beuerman RW, Kaufman HE (1999) Characterization of epithelial downgrowth by confocal microscopy. J Cataract Refract Surg 25: 1172-1174.

4. Al Saraiji H, Viestenz A, Hasenfus A, Schlötzer-Schrehardt U, Seitz B (2017) Block excision and tectonic corneoscleral graft for recurrent cystic epithelial downgrowth despite alcohol injection: Clinicopathologic report. JCRS Online Case Rep 5: 5-8.

5. Lenhart PD, Randleman JB, Grossniklaus HE, Stulting RD (2008) Confocal microscopic diagnosis of epithelial downgrowth. Cornea 27: 1138-1141.
6. Viestenz A, Küchle M, Naumann GO (2003) Block excision of epithelial ingrowth after cataract surgery--report on 15 patients. Clinical monthly sheets for ophthalmology 220: $465-470$.

7. Viestenz A, Seitz B, Viestenz A, Naumann GO (2018) Epithelial invasion after open globe injury. Clin Anat 31: 68-71.

8. Pai VC, Glasgow BJ (2010) MUC16 as a sensitive and specific marker for epithelial downgrowth. Arch Ophthalmol 128: 1407-1412.

9. Naumann G, Völcker HE (1975) Block-excision of intraocular processes. II. Epithelial ingrowth into the anterior segment of the eye. Clinical monthly sheets for ophthalmology 166: 448-457.

10. Kim SK, Ibarra MS, Syed NA, Sulewski ME, Orlin SE (2005). Development of epithelial downgrowth several decades after intraocular surgery. Cornea 24: 108-109.

11. Nemi A, Bahadur RP, Randleman JB (2008) Traumatic epithelial downgrowth after radial keratotomy. J Cataract Refract Surg 34: 327-329.

12. Aldave AJ, Hollander DA, Branco B, Crawford B, Abbott RL (2005) Primary graft failure associated with epithelial downgrowth: a case report. BMC ophthalmol 5: 11.

13. Reinhart WJ, Musch DC, Jacobs DS, Lee WB, Kaufman SC, et al. (2011) Deep anterior lamellar keratoplasty as an alternative to penetrating keratoplasty: A report by the American Academy of Ophthalmology. Ophthalmol 118: 209-218.

14. Seitz B, Szentmáry N, Langenbucher A, Hager T, Viestenz A, et al. (2016) Perforating keratoplasty for advanced keratoconus - from the hand / motor trepan up to the excimer laser and back to the femtosecond laser. Clinical monthly sheets for ophthalmology 233: 727-736.

Copyright: (C2020 Majkutova B. This is an open-access article distributed under the terms of the Creative Commons Attribution License, which permits unrestricted use, distribution, and reproduction in any medium, provided the original author and source are credited. 